PROCEEDINGS OF THE

AMERICAN MATHEMATICAL SOCIETY

Volume 135, Number 3, March 2007, Pages 795-803

S 0002-9939(06)08577-7

Article electronically published on September 11, 2006

\title{
WEIL-PETERSSON ISOMETRIES VIA THE PANTS COMPLEX
}

\author{
JEFFREY BROCK AND DAN MARGALIT
}

(Communicated by Richard A. Wentworth)

\begin{abstract}
We extend a theorem of Masur-Wolf which states that given a finite-area hyperbolic surface $S$, every isometry of the Teichmüller space for $S$ with the Weil-Petersson metric is induced by an element of the mapping class group for $S$. Our argument handles the previously untreated cases of the four times-punctured sphere, the once-punctured torus, and the twice-punctured torus.
\end{abstract}

\section{INTRODUCTION}

The purpose of this paper is to fill in several gaps in the understanding of WeilPetersson isometries. Throughout, $S$ is an orientable, finite-area hyperbolic surface, and $S_{g, n}$ denotes a surface of genus $g$ with $n$ punctures. The extended mapping class group for $S$ is

$$
\operatorname{Mod}(S)=\pi_{0}\left(\text { Homeo }^{ \pm}(S)\right)
$$

Let T $(S)$ denote the Teichmüller space of $S$ with the Weil-Petersson metric. MasurWolf proved that for $S \notin\left\{S_{1,1}, S_{0,4}, S_{1,2}\right\}$, every isometry of $\mathrm{T}(S)$ is induced by an element of $\operatorname{Mod}(S)$ [18. Their strategy is to show that an isometry of $\mathrm{T}(S)$ gives rise to an automorphism of the complex of curves for $S$. They then apply the theorem that, for exactly the surfaces in question, every automorphism of the complex of curves is induced by $\operatorname{Mod}(S)$; this is a theorem of Ivanov [12, with special cases due to Korkmaz and Luo [14, 15]. See Section 2 for definitions.

In this paper we take a slightly different viewpoint on the reasoning of MasurWolf to extend their result to all hyperbolic surfaces. Our shift in perspective involves the observation that an isometry $I$ of $\mathrm{T}(S)$ determines an automorphism $\bar{I}_{\star}$ of the pants complex for $S$. By a theorem of the second author, $\bar{I}_{\star}$ is necessarily induced by a mapping class $f$. It then follows from a theorem of Wolpert, which builds upon a theorem of the first author, that $f$ induces $I$; we remark that this theorem was a central step in Wolpert's own recasting of the Masur-Wolf result 25. We thus have the following theorem.

Received by the editors January 18, 2005 and, in revised form, October 14, 2005

2000 Mathematics Subject Classification. Primary 32G15; Secondary 32M99.

Key words and phrases. Teichmüller space, Weil-Petersson metric, isometries, mapping class groups.

The first author was partially supported by NSF grant number 0354288 .

The second author was partially supported by an NSF postdoctoral fellowship and a VIGRE postdoctoral position under NSF grant number 0091675 to the University of Utah.

(C)2006 American Mathematical Society 

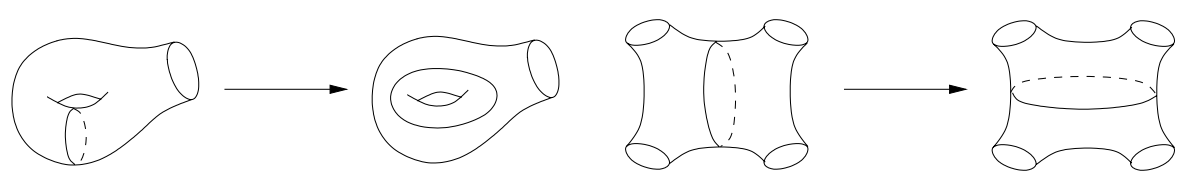

FiguRE 1. Elementary moves.

Main Theorem. The natural map $\eta: \operatorname{Mod}(S) \rightarrow \operatorname{Isom}(\mathrm{T}(S))$ is surjective. Further, $\operatorname{ker}(\eta) \cong \mathbb{Z}_{2}$ for $S \in\left\{S_{1,1}, S_{1,2}, S_{2,0}\right\}, \operatorname{ker}(\eta) \cong \mathbb{Z}_{2} \oplus \mathbb{Z}_{2}$ for $S=S_{0,4}$, $\operatorname{ker}(\eta)=\operatorname{Mod}(S)$ for $S=S_{0,3}$, and $\operatorname{ker}(\eta)=1$ otherwise.

It follows from the definition of the Weil-Petersson metric that $\operatorname{Mod}(S)$ acts by isometries; the content is that all isometries arise in this way.

The application of curve complexes to the study of isometries of Teichmüller space first appears in Ivanov's proof of Royden's Theorem, which is the analogue of our Main Theorem for the Teichmüller metric [13, 20].

\section{Preliminaries}

We briefly recall the basic definitions and concepts.

Complex of curves. The complex of curves $\mathrm{C}(S)$ for $S$, defined by Harvey [8], is the abstract simplicial flag complex with vertices corresponding to isotopy classes of essential simple closed curves in $S$, and edges between vertices that can be realized disjointly in $S$.

For our purposes, this complex has an undesirable property when $S$ is $S_{1,1}, S_{0,4}$, or $S_{1,2}$ : there are automorphisms of $\mathrm{C}(S)$ which are not induced by elements of $\operatorname{Mod}(S)$. For $S \in\left\{S_{1,1}, S_{0,4}\right\}$, this is because, as defined, $\mathrm{C}(S)$ is a countable discrete set of vertices with no edges.

The problem with $S_{1,2}$ is more subtle. Luo noticed that if $\iota$ is the hyperelliptic involution of $S_{1,2}$, then the projection $\pi: S_{1,2} \rightarrow S_{1,2} / \iota$ gives an isomorphism between $\mathrm{C}\left(S_{1,2}\right)$ and $\mathrm{C}\left(S_{0,5}\right)$. To see the isomorphism, one must identify $S_{0,5}$ with the complement of the cone points in $S_{1,2} / \iota$. One must also apply the fact, due to Birman and Viro, that $\iota$ acts trivially on $\mathrm{C}\left(S_{1,2}\right)$ [2], 22]. Since $\operatorname{Mod}\left(S_{0,5}\right)$ acts transitively on the vertices of $\mathrm{C}\left(S_{0,5}\right)$ (every curve has two punctures on one side and three on the other), it follows that some automorphisms of $\mathrm{C}\left(S_{1,2}\right)$ fail to preserve the set of vertices corresponding to separating curves. Such automorphisms clearly cannot arise from mapping classes (see [15]).

Pants complex. The pants complex $\mathrm{P}(S)$ for $S$, introduced by Hatcher and Thurston 10], 9], is the simplicial complex with vertices corresponding to pants decompositions of $S$ (i.e. maximal simplices of $\mathrm{C}(S)$ ), and edges connecting pants decompositions which differ by an elementary move, by which we mean that the two pants decompositions differ by only one curve, and the differing curves have the smallest possible geometric intersection number. The minimal intersection number is 1 or 2, depending on whether the curve being replaced lies in a single pair of pants or is the boundary between two pairs of pants; see Figure 1.

We will apply the following theorem of the second author [16]. We use $\operatorname{Aut}(\mathrm{P}(S))$ to denote the simplicial automorphism group of $\mathrm{P}(S)$. 
Theorem 2.1. The natural map $\theta: \operatorname{Mod}(S) \rightarrow \operatorname{Aut}(\mathrm{P}(S))$ is surjective. Further, $\operatorname{ker}(\theta) \cong \mathbb{Z}_{2}$ for $S \in\left\{S_{1,1}, S_{1,2}, S_{2,0}\right\}, \operatorname{ker}(\theta) \cong \mathbb{Z}_{2} \oplus \mathbb{Z}_{2}$ for $S=S_{0,4}, \operatorname{ker}(\theta)=$ $\operatorname{Mod}(S)$ for $S=S_{0,3}$, and $\operatorname{ker}(\theta)=1$ otherwise.

The proof of Theorem 2.1 is based on the aforementioned theorem of Ivanov, Korkmaz, and Luo, which gives an analogous statement for $\mathrm{C}(S)$. We remark that the term "pants complex" is often reserved for a particular simply-connected 2complex obtained by adding 2-cells to the graph defined above [9]. Theorem 2.1 holds for the graph as well as the 2-complex.

Teichmüller space. A point in the Teichmüller space $\mathrm{T}(S)$ of $S$ is given by a pair $(X, f)$, where $X$ is a finite-area hyperbolic surface $X$ and $f: S \rightarrow X$ is a homeomorphism. Two points $(X, f)$ and $(Y, g)$ are equivalent if $g \circ f^{-1}$ is isotopic to an isometry.

We note that $\mathrm{T}\left(S_{0,3}\right)$ is a single point, and in this case our main result is trivial.

Weil-Petersson metric. A point in $\mathrm{T}(S)$ is naturally a Riemann surface via its uniformization as a quotient of $\mathbb{H}^{2}$ by a Fuchsian group. In this way, the cotangent space $T_{X}^{\star}(\mathrm{T}(S))$ at a point $X$ is identified with the space of holomorphic quadratic differentials on $X$ (holomorphic forms of type $\phi(z) d z^{2}$, with simple poles at the punctures of $X$ ), which has the $L^{2}$ inner product defined by

$$
\langle\phi, \psi\rangle=\int_{X} \frac{\phi \bar{\psi}}{\rho^{2}} .
$$

Here, $\rho(z)|d z|$ is the hyperbolic metric on $X$. Then, the Weil-Petersson metric is defined by the pairing

$$
\langle\mu, \phi\rangle=\int_{X} \mu \phi,
$$

where $\phi \in T_{X}^{\star}(\mathrm{T}(S))$ and $\mu$ is an element of the tangent space $T_{X}(\mathrm{~T}(S))$, which is identified with the space of harmonic Beltrami differentials on $X$ (forms of type $\mu(z) \overline{d z} / d z ;$ see e.g. [11, [19]).

Chu and Wolpert showed that the Weil-Petersson metric is not complete [6], [23].

Augmented Teichmüller space. Masur gave an interpretation of points in the completion of $\mathrm{T}(S)$ as marked noded Riemann surfaces [17. A noded Riemann surface is a complex space with at most isolated singularities, called nodes, each possessing a neighborhood biholomorphic to a neighborhood of $(0,0)$ in the curve $\{z w=0\}$ in $\mathbb{C}^{2}$. Removing the nodes of a noded Riemann surface $W$ yields a (possibly disconnected) Riemann surface whose components (which are required to have negative Euler characteristic) we call the pieces of $W$. The notion of a noded Riemann surface was developed by Bers [1].

Given a simplex $\sigma$ of $\mathrm{C}(S)$, a marked noded Riemann surface $(W, f)$ with nodes at $\sigma$ is a noded Riemann surface $W$ equipped with a continuous mapping

$$
f: S \rightarrow W
$$

so that $\left.f\right|_{S \backslash \sigma}$ is a homeomorphism to the pieces of $W$. Two marked noded Riemann surfaces $(W, f)$ and $(Z, g)$ are equivalent if there is a continuous marking-preserving map $\phi: W \rightarrow Z$ which preserves nodes and is biholomorphic on the pieces. 
To describe a neighborhood of a point $(W, f)$ in Masur's completion of $\mathrm{T}(S)$, which we denote $\overline{\mathrm{T}(S)}$, we give coordinates adapted to the simplex $\sigma$. Given a maximal simplex $\tau$ with $\sigma \subset \tau$, there are Fenchel-Nielsen coordinates

$$
\left\{(\ell, \theta)_{\alpha}\right\} \in\left(\mathbb{R}_{>0} \times \mathbb{R}\right)^{|\tau|}
$$

for $\mathrm{T}(S)$ specifying length and twist parameters along each closed geodesic $\alpha$ determined by the vertices of $\tau$ (see, e.g., 11]). Then the extended Fenchel-Nielsen coordinates for $\tau$ are obtained by allowing lengths to range in $\mathbb{R}_{\geq 0}$ and stipulating that for any twist parameters $\theta$ and $\theta^{\prime}$ we have $(0, \theta)_{\alpha} \sim\left(0, \theta^{\prime}\right)_{\alpha}$.

The extended Fenchel-Nielsen coordinates for $(W, f)$, then, are given by setting the length parameters corresponding to the curves of $\sigma$ to 0 , and setting the other length and twist parameters equal to the corresponding Fenchel-Nielsen coordinates for the piece in which the geodesic lies. Then a neighborhood of $(W, f)$ is given by all surfaces in $\overline{\mathrm{T}(S)}$ with extended Fenchel-Nielsen coordinates close to those of $(W, f)$. We will in practice refer to $(W, f)$ as a noded surface with curves in $\sigma$ pinched.

From this description of points in the completion, and the fact that $\mathrm{T}(S)$ is homeomorphic to a ball, we see that a point in $\overline{\mathrm{T}(S)}$ has a compact neighborhood if and only if it is a point of $\mathrm{T}(S)$.

Stratification. We think of $\overline{\mathrm{T}(S)}$ as a stratified space, with $\mathcal{F}_{k}$ denoting the stratum consisting of surfaces with $k$ curves pinched. Also, $\mathcal{F}$ denotes $\overline{\mathrm{T}(S)}-\mathrm{T}(S)$. Points in the stratum corresponding to maximally noded surfaces are naturally associated with the pants decompositions that are pinched. These points are isolated from each other, since there is a unique hyperbolic structure on $S_{0,3}$.

As Teichmüller spaces themselves, each connected component $\mathcal{O}$ of $\mathcal{F}_{k}$ comes equipped with its own Weil-Petersson metric $d_{\mathcal{O}}$. On the other hand, $\mathcal{O}$ inherits a metric $\left.d_{\mathrm{T}(S)}\right|_{\mathcal{O}}$ as the completion of $\mathrm{T}(S)$. Masur proved that the Weil-Petersson metric tensor on $\mathrm{T}(S)$ extends continuously to the intrinsic Weil-Petersson metric tensor on strata in $\mathcal{F}$ [17, and Daskalopoulos-Wentworth and Wolpert proved that length minimizing paths in $\mathcal{F}_{k}$ do not enter $\mathrm{T}(S)$ [7, 24]. Together these facts give the following (see [18, Lem. 1.3.1], 7], 24]).

Theorem 2.2. On any connected component $\mathcal{O}$ of $\mathcal{F}_{k}$, the metrics $d_{\mathcal{O}}$ and $\left.d_{\mathrm{T}(S)}\right|_{\mathcal{O}}$ are the same.

Negative curvature. By work of Royden, Tromba, and Wolpert, the Weil-Petersson metric has negative sectional curvatures and is geodesically convex 21, 24. It then follows from general principles that $\overline{\mathrm{T}(S)}$ is a $\operatorname{CAT}(0)$ metric space (see [3]).

Visual sphere. Each point $X$ in $\mathrm{T}(S)$ has a visual sphere $\mathcal{V}_{X}(S)$, which is the unit tangent space at $X$. Due to the non-completeness of the Weil-Petersson metric, certain directions in the visual sphere correspond to finite-length geodesics emanating from $X$ that leave every compact subset of $\mathrm{T}(S)$. These finite rays terminate at noded Riemann surfaces in $\mathcal{F}$.

As a consequence of the $\operatorname{CAT}(0)$ property for $\overline{\mathrm{T}(S)}$, the first author proved the following density theorem [4].

Theorem 2.3. For any $S$ and any $X \in \mathrm{T}(S)$, the finite rays are dense in the visual sphere $\mathcal{V}_{X}(S)$. 
The idea is that every point $X$ of $\mathrm{T}(S)$ is within a uniformly bounded distance of a maximally noded surface $N(X)$ (see [5]). Thus, given a sequence of points $X_{n}$ diverging from $X$ along an infinite ray from $X$, a sequence of points of $\mathcal{V}_{X}(S)$ corresponding to the directions determined by the geodesics joining $X$ to $N\left(X_{n}\right)$ converges to the given infinite ray. Wolpert used Theorem 2.3 to prove the following theorem [25, which immediately implies that a Weil-Petersson isometry is determined by its action on the maximally noded surfaces.

Theorem 2.4. $\overline{\mathrm{T}(S)}$ is the closed convex hull of its maximally noded surfaces.

Wolpert has in fact already used this corollary to give a simplification of the proof of Masur-Wolf (but not our extension) [25].

\section{EDGE STRATA}

In this section, we study the stratum of $\overline{\mathrm{T}(S)}$ where all but one of the curves of a pants decomposition are pinched; in Lemma 4.2, this stratum is where we will be able to see the edges of $\mathrm{P}(S)$. By Theorem 2.2 each connected component of this stratum is isometric to $\mathrm{T}(R)$ for $R$ equal to either $S_{1,1}$ or $S_{0,4}$.

In either case, we have that $\mathrm{T}(R)$ is topologically $\mathbb{H}^{2}$, and $\operatorname{Mod}(R) / Z \cong \mathrm{PGL}_{2}(\mathbb{Z})$, where $Z$ is the center of $\operatorname{Mod}(R)\left(Z=\mathbb{Z}_{2}\right.$ if $R=S_{1,1}$, and $Z=\mathbb{Z}_{2} \oplus \mathbb{Z}_{2}$ if $\left.R=S_{0,4}\right)$. The action of $\operatorname{Mod}(R)$ on $\mathrm{T}(R)$ then coincides with the usual action of $\mathrm{PGL}_{2}(\mathbb{Z})$ on $\mathbb{H}^{2}$ by Möbius transformations and complex conjugation.

Also, $\mathcal{F}=\overline{\mathrm{T}(R)}-\mathrm{T}(R)$ is the set of rational points of $\partial \mathbb{H}^{2}$, and $\overline{\mathrm{T}(R)}$ has the horoball topology. That $\mathcal{F}$ is discrete agrees with the fact that any noded surface is maximally noded (pants decompositions consist of exactly one curve). In this way, isotopy classes of simple closed curves in $R$ can be identified with $\mathbb{Q} \cup\{1 / 0\}$, and two such isotopy classes corresponding to the reduced fractions $p / q$ and $r / s$ are connected by an edge in $\mathrm{P}(R)$ exactly when $|p s-q r|=1$.

3.1. Farey graph. Let $\mathcal{G}$ denote the usual embedding of the Farey graph in $\mathbb{H}^{2}$ : in the upper half-plane model of $\mathbb{H}^{2}$, its vertices are at rational points (including $\infty)$, and its edges are hyperbolic geodesics connecting (reduced) rational numbers $p / q$ and $r / s$ whenever $|p s-q r|=1$ (see Figure 2). We will now see that each edge of $\mathcal{G}$ is a Weil-Petersson geodesic.

The imaginary axis is a geodesic, since it is the fixed set of the isometry given by a mapping class corresponding to

$$
\left(\begin{array}{rr}
1 & 0 \\
0 & -1
\end{array}\right)
$$

which acts on $\mathbb{H}^{2}$ (in the upper half-plane model) by $z \mapsto-\bar{z}$. The $\mathrm{PGL}_{2}(\mathbb{Z}$ )-orbit of this geodesic is $\mathcal{G}$. Thus, $\mathcal{G}$ gives a geodesic between any noded surfaces whose corresponding pants decompositions have distance 1 in $\mathrm{P}(R)$.

3.2. Weil-Petersson vs. pants distance. We will now show that the WeilPetersson metric encodes adjacency in the pants complex $\mathrm{P}(R)$.

In the following lemma, we denote a (geodesic) triangle, and any of its edges, by a set of vertices. A triangle is called a tripod if any edge is contained in the union of the other two edges. 


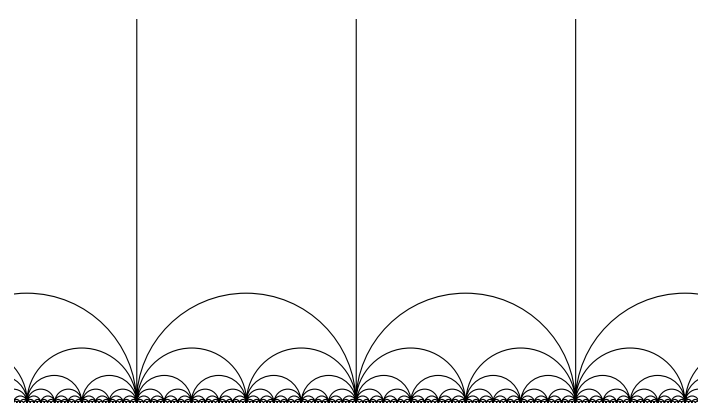

Figure 2. The Farey graph $\mathcal{G}$ in the upper half-plane.

Lemma 3.1. Let $(X, d)$ be a $\mathrm{CAT}(0)$ metric space, and let $A B C$ be an equilateral triangle in $X$ which is not a tripod, and which has side length $L$. If $D$ is any point on $B C$, then $d(A, D)>L / 2$.

Proof. Assume that, say, $d(B, D) \leq L / 2$. The triangle inequality implies that $d(A, D) \geq L / 2$ and that if $d(A, D)=L / 2$, then $d(B, D)=d(C, D)=L / 2$. In the latter case, the uniqueness of geodesics in $\mathrm{CAT}(0)$ spaces implies that $A D C=A C$ and $A D B=A B$; thus, $A B C$ is a tripod.

For the next lemma, $L$ is the Weil-Petersson length of edges of $\mathcal{G}, \mathrm{d}_{\mathrm{P}}$ denotes combinatorial distance in $\mathrm{P}(R)$, and $\mathrm{d}_{\mathrm{WP}}$ denotes Weil-Petersson distance.

Lemma 3.2. If $P$ and $P^{\prime}$ are pants decompositions of $R$ with $\mathrm{d}_{\mathrm{P}}\left(P, P^{\prime}\right)>1$, then the corresponding noded surfaces $W$ and $W^{\prime}$ in $\mathrm{T}(R)$ have $\mathrm{d}_{\mathrm{WP}}\left(W, W^{\prime}\right)>L$.

Proof. Let $g$ be the geodesic from $W$ to $W^{\prime}$. Since $\mathrm{d}_{\mathrm{P}}\left(P, P^{\prime}\right)>1, g$ passes through at least two triangles of $\mathcal{G}$, and this gives a natural division of $g$ into segments. By Lemma 3.1, the segments containing $W$ and $W^{\prime}$ each have length greater than $L / 2$, so $\mathrm{d}_{\mathrm{WP}}\left(W, W^{\prime}\right)>L$.

Remark. $L$ has different values, say $L_{S_{1,1}}$ and $L_{S_{0,4}}$, depending on the surface. In fact, $L_{S_{0,4}}=2 L_{S_{1,1}}$, and more generally the metrics for $\mathrm{T}\left(S_{0,4}\right)$ and $\mathrm{T}\left(S_{1,1}\right)$ differ by the same factor. To see this, note that for $R$ either $S_{1,1}$ or $S_{0,4}$, the quotient of $R$ by $Z(\operatorname{Mod}(R))$ is a genus zero orbifold with one cusp and three cone points of order 2. Since $\pi: R \rightarrow R / Z(\operatorname{Mod}(R))$ is a local isometry, and $\pi$ gives a canonical bijection between $\mathrm{T}(R)$ and $\mathrm{T}(R / Z(\operatorname{Mod}(R)))$, it follows from the definition that the metric on $\mathrm{T}(R)$ is the metric on $\mathrm{T}(R / Z(R))$ multiplied by the degree of $\pi$. This fact, however, is not needed for the proof.

\section{Proof of the Main Theorem}

Let $S$ be any surface, other than $S_{0,3}$, with negative Euler characteristic, let $I \in \operatorname{Isom}(\mathrm{T}(S))$, and let $\bar{I}$ be the natural extension of $I$ to the completion $\overline{\mathrm{T}(S)}$. We will use $\mathcal{F}_{\geq k}$ to denote $\bigcup_{i \geq k} \mathcal{F}_{i}$.

The following lemma is also proven by Masur-Wolf [18, Lem. 1.3.4, Lem. 1.3.5]; however, the proof given here is more elementary, relying only on topology and not at all on curvature. The lemma also follows from earlier work of Wolpert, who proved that the connected components of strata are characterized as being connected unions of open geodesics [24, Thm. 13]. 
Lemma 4.1. $\bar{I}(\mathcal{F}) \subset \mathcal{F}, I$ and $\bar{I}$ are surjective, and $\bar{I}$ preserves strata.

Proof. For the first statement, suppose that $\bar{I}(W) \in \mathrm{T}(S)$. Then $\bar{I}(W)$ has a compact neighborhood $K$. Let $K^{\prime}=K \cap \bar{I}(\overline{\mathrm{T}(S)})$. We have that $K^{\prime}$ is compact, since $\bar{I}(\overline{\mathrm{T}(S)})$ is closed (it is an isometric embedding of a complete space). But this implies that $W$ has a compact neighborhood (namely $\bar{I}^{-1}\left(K^{\prime}\right)$ ), and so $W \in \mathrm{T}(S)$.

For surjectivity of $I$, it suffices to show that $I$ is proper (any proper embedding of a manifold without boundary into itself is surjective). Indeed, let $K$ be a compact subset of $\mathrm{T}(S)$. As above, $K^{\prime}=K \cap \bar{I}(\overline{\mathrm{T}(S)})$ is compact. Since $\bar{I}(\mathcal{F}) \subset \mathcal{F}$, $K^{\prime}=K \cap I(\mathrm{~T}(S))$. Hence, $I^{-1}\left(K^{\prime}\right)$ is compact. Thus, $I$ is surjective, and it immediately follows that $\bar{I}$ is surjective.

For the last statement, we inductively show that $W \in \mathcal{F}_{k}$ if and only if $\bar{I}(W) \in$ $\mathcal{F}_{k}$. The base case is $\mathcal{F}_{0}=\mathrm{T}(S)$ : by definition, $\bar{I}(W) \in \mathrm{T}(S)$ whenever $W \in \mathrm{T}(S)$, and we have just shown that $\bar{I}(\mathcal{F}) \subset \mathcal{F}$.

Now suppose $\bar{I}$ preserves $\mathcal{F}_{i}$ for $0 \leq i \leq k-1$. Then $\bar{I}$ restricts to an isometry of $\mathcal{F}_{\geq k}$, which is a homeomorphism since $\bar{I}$ is surjective. The points of $\mathcal{F}_{k}$ are characterized by having compact neighborhoods in $\mathcal{F}_{\geq k}$. Since $\left.\bar{I}\right|_{\mathcal{F}_{\geq k}}$ is a homeomorphism, $\mathcal{F}_{k}$ is preserved.

We now combine Lemmas 3.2 and 4.1 to bridge the gap between isometries of $\mathrm{T}(S)$ and automorphisms of $\mathrm{P}(S)$. In the proof, we use $\mathcal{F}_{M}$ to denote the highest level stratum (the 0 -dimensional stratum) of $\mathrm{T}(S)$. Also, each connected component $\mathcal{O}$ of $\mathcal{F}_{M-1}$ (the 2-dimensional stratum) comes equipped with a Farey graph, which we denote by $\mathcal{G}_{\mathcal{O}}$.

Lemma 4.2. $\bar{I}$ induces $\bar{I}_{\star} \in \operatorname{Aut}(\mathrm{P}(S))$.

Proof. Points of $\mathcal{F}_{M}$ correspond to vertices of $\mathrm{P}(S)$. Thus, by Lemma 4.1, we have that $\bar{I}$ induces an automorphism $\bar{I}_{\star}$ of the 0 -skeleton of $\mathrm{P}(S)$.

It remains to show that $\bar{I}_{\star}$ preserves edges of $\mathrm{P}(S)$. If $\mathcal{O}$ is any connected component of $\mathcal{F}_{M-1}$, then $\mathcal{O}$ and $\bar{I}(\mathcal{O})$ are both copies of either $\mathrm{T}\left(S_{1,1}\right)$ or $\mathrm{T}\left(S_{0,4}\right)$, and the edges of $\mathcal{G}_{\mathcal{O}}$ and $\mathcal{G}_{\bar{I}(\mathcal{O})}$ correspond to edges of $\mathrm{P}(S)$.

The Weil-Petersson lengths of the edges of $\mathcal{G}_{\mathcal{O}}$ and $\mathcal{G}_{\bar{I}(\mathcal{O})}$ must be the same, since, by Lemma 4.1, the smallest length of a geodesic connecting noded surfaces is an isometry invariant. By Lemma 3.2, Lemma 4.1, and Theorem 2.2, $\bar{I}\left(\mathcal{G}_{\mathcal{O}}\right)=\mathcal{G}_{\bar{I}(\mathcal{O})}$; but this is exactly saying that $\bar{I}_{\star} \in \operatorname{Aut}(\mathrm{P}(S))$.

We note that, in the proof, the surfaces corresponding to $\mathcal{O}$ and $\bar{I}(\mathcal{O})$ must be the same; this follows from the remark at the end of Section 3

We are now ready to prove the Main Theorem.

Proof. Let $I \in \operatorname{Isom}(\mathrm{T}(S))$. By Lemma 4.2, $I$ induces $\bar{I}_{\star} \in \operatorname{Aut}(\mathrm{P}(S))$. By Theorem 2.1, $\bar{I}_{\star}$ is induced by a mapping class $f$. By Theorem 2.4, $f$ induces $I$.

Also, by Theorem 2.4. the kernel of the map $\eta: \operatorname{Mod}(S) \rightarrow \operatorname{Isom}(\mathrm{T}(S))$ is the same as the kernel of $\theta: \operatorname{Mod}(S) \rightarrow \operatorname{Aut}(\mathrm{P}(S))$.

\section{ACKNOWLEDGEMENTS}

We would like to thank Benson Farb for suggesting the pants complex as a tool for understanding Weil-Petersson isometries. This paper came out of a minicourse hosted by the University of Utah. We would like to thank the participants, in particular Bob Bell and Ken Bromberg, for helpful discussions. The remark at 
the end of Section 3 came from discussions with Chris Leininger. Also, the proof of Lemma 4.1 presented here is due to Mladen Bestvina; we thank him, as well as Howard Masur, for many enlightening conversations. We are grateful to Indira Chatterji and Kevin Wortman for useful comments on earlier drafts of the paper. Finally, the referee's comments were especially helpful for improving the exposition.

\section{REFERENCES}

1. L. Bers, Spaces of degenerating Riemann surfaces, Discontinuous Groups And Riemann Surfaces, Annals Of Math Studies 76, Princeton University Press, 1974, pp. 43-55. MR0361051 (50:13497)

2. Joan S. Birman, Braids, links, and mapping class groups, Princeton University Press, Princeton, N.J., 1974, Annals of Mathematics Studies, No. 82. MR0375281 (51:11477)

3. Martin R. Bridson and André Haefliger, Metric spaces of non-positive curvature, Grundlehren der Mathematischen Wissenschaften [Fundamental Principles of Mathematical Sciences], vol. 319, Springer-Verlag, Berlin, 1999. MR.1744486 (2000k:53038)

4. Jeffrey F. Brock, The Weil-Petersson visual sphere.

5., The Weil-Petersson metric and volumes of 3-dimensional hyperbolic convex cores, J. Amer. Math. Soc. 16 (2003), no. 3, 495-535 (electronic). MR 1969203 (2004c:32027)

6. Tienchen Chu, The Weil-Petersson metric in the moduli space, Chinese J. Math. 4 (1976), no. 2, 29-51. MR0590105 (58:28683)

7. Georgios Daskalopoulos and Richard Wentworth, Classification of Weil-Petersson isometries, Amer. J. Math. 125 (2003), no. 4, 941-975. MR1993745 (2004d:32011)

8. W. J. Harvey, Geometric structure of surface mapping class groups, Homological group theory (Proc. Sympos., Durham, 1977), Cambridge Univ. Press, Cambridge, 1979, pp. 255-269. MR0564431 (82h:57012)

9. Allen Hatcher, Pierre Lochak, and Leila Schneps, On the Teichmüller tower of mapping class groups, J. Reine Angew. Math. 521 (2000), 1-24. MR.1752293(2001h:57018)

10. Allen Hatcher and William Thurston, A presentation for the mapping class group of a closed orientable surface, Topology 19 (1980), no. 3, 221-237. MR0579573 (81k:57008)

11. Y. Imayoshi and M. Taniguchi, An introduction to Teichmüller spaces, Springer-Verlag, Tokyo, 1992, Translated and revised from the Japanese by the authors. MR1215481 (94b:32031)

12. Nikolai V. Ivanov, Automorphism of complexes of curves and of Teichmüller spaces, Internat. Math. Res. Notices (1997), no. 14, 651-666. MR1460387 (98j:57023)

13. Isometries of Teichmüller spaces from the point of view of Mostow rigidity, Topology, ergodic theory, real algebraic geometry, Amer. Math. Soc. Transl. Ser. 2, vol. 202, Amer. Math. Soc., Providence, RI, 2001, pp. 131-149. MR1819186 (2001m:30057)

14. Mustafa Korkmaz, Automorphisms of complexes of curves on punctured spheres and on punctured tori, Topology Appl. 95 (1999), no. 2, 85-111. MR.1696431 (2000d:57025)

15. Feng Luo, Automorphisms of the complex of curves, Topology 39 (2000), no. 2, 283-298. MR 1722024 (2000j:57045)

16. Dan Margalit, Automorphisms of the pants complex, Duke Math. J. 121 (2004), no. 3, 457479. MR.2040283 (2004m:57037)

17. Howard Masur, Extension of the Weil-Petersson metric to the boundary of Teichmuller space, Duke Math. J. 43 (1976), no. 3, 623-635. MR0417456 (54:5506)

18. Howard Masur and Michael Wolf, The Weil-Petersson isometry group, Geom. Dedicata 93 (2002), 177-190. MR1934697 (2003j:32017)

19. Subhashis Nag, The complex analytic theory of Teichmüller spaces, Canadian Mathematical Society Series of Monographs and Advanced Texts, John Wiley \& Sons, Inc., New York, 1988, A Wiley-Interscience Publication. MR0927291 (89f:32040)

20. H. L. Royden, Automorphisms and isometries of Teichmüller space, Advances in the Theory of Riemann Surfaces (Proc. Conf., Stony Brook, N.Y., 1969), Ann. of Math. Studies, No. 66. Princeton Univ. Press, Princeton, N.J., 1971, pp. 369-383. MR0288254 (44:5452)

21. A. J. Tromba, On a natural algebraic affine connection on the space of almost complex structures and the curvature of Teichmüller space with respect to its Weil-Petersson metric, Manuscripta Math. 56 (1986), no. 4, 475-497. MR860734 (88c:32034) 
22. O. Ja. Viro, Links, two-sheeted branching coverings and braids, Mat. Sb. (N.S.) 87(129) (1972), 216-228. MR0298649 (45:7701)

23. Scott Wolpert, Noncompleteness of the Weil-Petersson metric for Teichmüller space, Pacific J. Math. 61 (1975), no. 2, 573-577. MR0422692 (54:10678)

24. Scott A. Wolpert, Geodesic length functions and the Nielsen problem, J. Differential Geom. 25 (1987), no. 2, 275-296. MR0880186 (88e:32032)

25. Geometry of the Weil-Petersson completion of Teichmüller space, Surveys in differential geometry, Vol. VIII (Boston, MA, 2002), Surv. Differ. Geom., VIII, Int. Press, Somerville, MA, 2003, pp. 357-393. MR2039996 (2005h:32032)

Department of Mathematics, Brown University, Box 1917, Providence, Rhode Island 02912

E-mail address: brock@math.brown.edu

Department of Mathematics, University of Utah, 155 S 1440 East, Salt Lake City, UTAн 84112-0090

E-mail address: margalit@math.utah.edu 